AperTO - Archivio Istituzionale Open Access dell'Università di Torino

\title{
Adalimumab trough levels predict Crohn's disease clinical course
}

\section{This is the author's manuscript}

Original Citation:

Availability:

This version is available http://hdl.handle.net/2318/1660639

since 2019-07-30T16:22:35Z

Published version:

DOI:10.1016/j.dld.2017.09.139

Terms of use:

Open Access

Anyone can freely access the full text of works made available as "Open Access". Works made available under a Creative Commons license can be used according to the terms and conditions of said license. Use of all other works requires consent of the right holder (author or publisher) if not exempted from copyright protection by the applicable law. 


\section{Adalimumab trough levels predict Crohn's disease clinical course}

Davide Giuseppe Ribaldone, MD1, Tilde Manetta, $\mathrm{PhD}^{2}$, Giulio Mengozzi, MD², Giorgio Maria Saracco, MD, $\mathrm{PhD}^{1}$, Rinaldo Pellicano, $\mathrm{MD}^{3}$, and Marco Astegiano, $\mathrm{MD}^{3}$

${ }^{1}$ Department of Medical Sciences, Division of Gastroenterology, University of Torino, Torino, Italy

2 Biochimical Laboratory, A.O.U. Città Della Salute E Della Scienza, Turin, Italy

${ }^{3}$ Department of General and Specialistic Medicine, Gastroenterologia-U, Città della Salute e della Scienza di Torino, C.so Bramante 88, 10126 Turin, Italy

Electronic word count (excluding abstract, references, tables, figures): 1591

Correspondence: Davide Giuseppe Ribaldone, Department of General and Specialistic Medicine, Gastroentrologia-U, Città della Salute e della Scienza di Torino, C.so Bramante 88, 10126 Turin, Italy; tel (0039)0116335208, fax (0039)0116336752,davrib_1998@yahoo.com

Conflicts of Interest and Source of Funding: none declared. 
Keywords: anti-TNF; biologics; Crohn disease; inflammatory bowel diseases. 
In Crohn's disease (CD) two third of patients have a clinical response to antiTNF, but less than $50 \%$ of the patients who respond to therapy maintain the response one year after the beginning of the anti-TNF therapy.

Part of the inability to maintain long-term remission is thought to result from the occurrence of antibody against adalimumab (AAA) and most of this loss of efficacy may be due to an inadequate optimization of the therapeutic dosage [1]. In case of relapse, an empiric adalimumab dosage increment in a patient with a high level of AAA or with adalimumab trough level in therapeutic range is unlikely useful and may expose the patient to damage resulting from over-therapeutic dose of the drug or to AAA-mediated adverse events. Identifying patients with subtherapeutic adalimumab trough levels allows to target the therapy to those patients who really need a different administration rate.

Till now there are no studies that prove the ability of trough adalimumab level and of AAA to predict the long-term disease behaviour in CD patients naïve to antiTNF.

We conducted a double blind (adalimumab trough levels and AAA had been available to the clinicians at the end of the study; the laboratory was blind to patient's clinical characteristics) prospective, monocenter, observational study in the "Città della Salute e della Scienza" hospital in Turin, Italy, between January 2016 and April 2017.

Inclusion criteria were age higher than 14 years old, CD diagnosed according to ECCO criteria [2], primary response to the adalimumab induction regimen, treatment with adalimumab (indication luminal CD) from at least 2 months, in a stable dose of $40 \mathrm{mg}$ every two week, naïve to other anti-TNF or adalimumab. The 
follow-up was concluded in case of a change in the dose or stop of adalimumab due to loss of efficacy, adverse drug reaction, complete response and in case of the need to start systemic corticosteroids.

The adalimumab trough levels and the AAA were randomly assessed, one time only per patient, the day before a next administration of the drug, together with Creactive protein (CRP) and fecal calprotectin. At the end of follow up the AAA trough levels and the CRP were again assessed. Clinical activity was assessed with Harvey-Bradshaw index [3].

The method used was the TNFa-Blocker ELISA kit (Immundiagnostik). For adalimumab trough levels dosing the Immundiagnostik TNFa-Blocker monitoring, adalimumab drug level ELISA (K9657) has been used. For the AAA dosage, the Immundiagnostik TNFa-Blocker ADA, TOTAL antibodies against adalimumab ELISA (K9651) has been used. These kits allow the measurement of the AAA even if the adalimumab is present in the patient serum.

The primary outcomes were 1) the adalimumab random trough level predictive ability of disease activity and of CRP level at the end of follow up, calculating the ROC curve with the optimum sensitivity and specificity value; 2) the AAA random trough level predictive ability of disease activity and of CRP at the end of follow up; 3) the adalimumab random trough level predictive ability of the AAA onset at the end of follow up.

The secondary outcomes were 1) the association between the adalimumab random trough level and the disease activity, CRP, fecal calprotectin in that moment; 2) the association between the AAA random trough level and the disease activity, CRP, fecal calprotectin in that moment; 3) the influence of azathioprine combination therapy on the adalimumab and the AAA trough levels. 
The statistical significance level was set to $95 \%$. The statistical analysis was performed by using MedCalc software (version 14.8.1).

In the study period $40 \mathrm{CD}$ currently treated with adalimumab, naïve to other antiTNF, patients were included. The clinical characteristics of included patients are reported in Table 1.

Table 1

The average follow-up was 10 months (range $3-14$ months).

At the time of the assessment, 28 of $40(70 \%)$ patients were in remission or in mild disease activity $(\mathrm{HBI}<=7), 12$ of $35(30 \%)$ were in moderate or severe disease activity $(\mathrm{HBI}>=8)$.

No adverse reaction to adalimumab administration occurred.

An adalimumab trough level $>0.65[\mu \mathrm{g} / \mathrm{ml}]$ predicted remission / mild disease activity $(\mathrm{HBI}<=7) 10$ months after the dosage with an AUC $=0.85(\mathrm{P}<0.0001)$ $($ sensitivity $=80 \%$, specificity $=90 \%)($ Figure 1$)$.

Figure 1

An adalimumab trough level $>1.8[\mu \mathrm{g} / \mathrm{ml}]$ predicted a negative CRP 10 months after the adalimumab trough level dosage with an AUC $=0.85(P<0.0001)$ (sensitivity $=85.7 \%$, specificity $=78.3 \%$ ). 
An AAA trough level $<=0.1[\mathrm{AU} / \mathrm{ml}]$ predicted remission / mild disease activity 10 months after the dosage with an AUC $=0.82(P=0.0001)$ (sensitivity $=80 \%$, specificity $=85.7 \%)($ Figure 2$)$.

Figure 2

An AAA trough level $<=1.1[\mathrm{AU} / \mathrm{ml}]$ predicted a negative CRP 10 month after the AAA trough level dosage with an AUC $=0.84(P=0.0002)$ (sensitivity $=85.7 \%$, specificity $=82.6 \%)$.

14 of 40 patients (35\%) have developed an AAA trough level $>0.1[\mathrm{AU} / \mathrm{ml}]$, at a median time of 13 months from the beginning of adalimumab therapy.

In all patients that developed an AAA trough level $>0.1[\mathrm{AU} / \mathrm{ml}]$, the adalimumab trough level had fallen under the threshold of $1.9[\mu \mathrm{g} / \mathrm{ml}]$; no patients that maintained the adalimumab trough level over this threshold had developed AAA at the end of follow up

The increasing of time in adalimumab therapy did not correlate with the development of anti-adalimumab antibody $(P=0.75)$.

In patients with a $\mathrm{CD}$ in remission or mild disease activity $(\mathrm{HBI}<=7)$ at the time of the random dosage, the median adalimumab trough level resulted 9.4 $[\mu \mathrm{g} / \mathrm{ml}]$, versus a median adalimumab trough level of $0.5[\mu \mathrm{g} / \mathrm{ml}]$ in patients with a $\mathrm{CD}$ in moderate or severe disease activity $(\mathrm{HBI}>=8)(P=0.0007)$

A declining of adalimumab trough level correlated with higher level, at that time, of CRP (correlation coefficient $=-0.64, \mathrm{P}=0.0002,95 \%$ C.I. $=-0.81--0.35$ ) 
and of calprotectin (correlation coefficient $=-0.64, \mathrm{P}=0.0002,95 \%$ C.I. $=-0.65--$ $0.26)$.

In patients with a $\mathrm{CD}$ in remission or mild disease activity $(\mathrm{HBI}<=7)$ at the time of the random dosage, the median AAA trough level resulted $0.1[\mathrm{AU} / \mathrm{ml}$, versus a median AAA trough level of $5[\mathrm{AU} / \mathrm{ml}]$ in patients with a $C D$ in moderate or severe disease activity $(P=0.002)$

An increasing of AAA trough level correlated with higher level, at that time, of CRP (correlation coefficient $=0.8, P<0.0001$, I.C. $95 \%=0.61-0.9$ ) and of calprotectin (correlation coefficient $=0.7, \mathrm{P}=<0.0001$, I.C. $95 \%=0.45-0.85$ ).

The adalimumab - azathioprine combination therapy did not change the adalimumab median trough levels at the time of the random dosage $(8[\mu \mathrm{g} / \mathrm{ml}]$ in monotherapy group, $4.5[\mu \mathrm{g} / \mathrm{ml}]$ in combination therapy group, $\mathrm{P}=0.47$ ).

The adalimumab - azathioprine combination therapy did not change the AAA median trough levels at the time of the random dosage $(0.1[\mathrm{AU} / \mathrm{ml}]$ in monotherapy group, $2.6[\mathrm{AU} / \mathrm{ml}]$ in combination therapy group, $P=0.31)$.

By comparing our findings with those of the literature, in a multicentre, retrospective, cohort study an adalimumab threshold trough level of $3[\mu \mathrm{g} / \mathrm{ml}]$ best differs the presence of absence of inflammation in that moment [4]. In a retrospective study on $30 \mathrm{CD}$ patients experience to infliximab, the presence of AAA was related with a primary failure to adalimumab [5]. In an observational, prospective, monocenter study about 168 CD patients with a secondary failure to infliximab, the patients with AAA at the end of follow up had a lower adalimumab trough level at 4 weeks of therapy [6]. A combination therapy with azathioprine did not change the therapeutic results. In a monocenter study about $40 \mathrm{CD}$ patients treated with adalimumab maintenance therapy, the adalimumab trough levels were significantly 
elevated in patients with inactive disease compared to those with active disease. A combination therapy with azathioprine did not change the adalimumab trough level [7]. In a transversal, multicentre study about $71 \mathrm{CD}$ patients at ROC curve resulted a positive correlation between adalimumab trough level and the remission in that moment. There wasn't correlation between adalimumab trough level and a combination therapy with azathioprine. The AAA level did not differentiate an active disease [8]. In a prospective, observational cohort study about 23 CD patients, the patients with a disease in remission had higher trough adalimumab level respect to patients with a mild activity of disease and to patients with moderate or severe activity of disease. The median adalimumab trough level resulted statistically lower in patients with AAA than in patients without [9]. In a retrospective cross-sectional study of 67 with IBD treated with adalimumab the authors [10] found a median serum levels of adalimumab significantly higher in patients with mucosal healing than in patients with active disease.

Our is the first double blind, prospective study in $C D$ patients treated with adalimumab, naive to other anti-TNF that demonstrates the ability of a random adalimumab and AAA trough level to predict a well-controlled disease at almost one year from the dosage.

Consistent with the clinical disease activity, the fact that a statistically significant negative correlation was found between the adalimumab trough levels and the CRP and the calprotectin at the time of the biological drug dosing, shows that a low serum drug level is insufficient to control the inflammation.

In agreement with all the studies reported in literature, there is not a statistically significant difference between the adalimumab median trough levels or the AAA median trough levels in patients in combination therapy with azathioprine. 
Further investigation in a large cohort and a correlation with the mucosal healing would be of benefit.

\section{References}

1. Vaughn BP, Sandborn WJ, Cheifetz AS. Biologic concentration testing in inflammatory bowel disease. Inflamm Bowel Dis 2015;21:1435-42.

2. Gomollón F, Dignass A, Annese V, et al. 3rd European Evidence-based Consensus on the Diagnosis and Management of Crohn's Disease 2016: Part 1: Diagnosis and Medical Management. J Crohns Colitis 2017;11:3-25.

3. Harvey RF, Bradshaw JM. A simple index of Crohn's-disease activity. Lancet 1980;1:514.

4. Yanai $\mathrm{H}$, Lichtenstein L, Assa $\mathrm{A}$, et al. Levels of drug and antidrug antibodies are associated with outcome of interventions after loss of response to infliximab or adalimumab. Clin Gastroenterol Hepatol 2015;13:522-30.e2.

5. West RL, Zelinkova Z, Wolbink GJ, et al. Immunogenicity negatively influences the outcome of adalimumab treatment in Crohn's disease. Aliment Pharmacol Ther 2008;28:1122-6.

6. Adedokun OJ, Sandborn WJ, Feagan BG, et al. Association between serum concentration of infliximab and efficacy in adult patients with ulcerative colitis. Gastroenterology 2014;147:1296-1307.e5.

7. Imaeda $\mathrm{H}$, Takahashi $\mathrm{K}$, Fujimoto $\mathrm{T}$, et al. Clinical utility of newly developed immunoassays for serum concentrations of adalimumab and anti-adalimumab antibodies in patients with Crohn's disease. J Gastroenterol 2014;49:100-9. 
8. Mazor $\mathrm{Y}$, Almog R, Kopylov $\mathrm{U}$, et al. Adalimumab drug and antibody levels as predictors of clinical and laboratory response in patients with Crohn's disease. Aliment Pharmacol Ther 2014;40:620-8.

9. Bodini G, Giannini EG, Savarino V, et al. Adalimumab trough serum levels and anti-adalimumab antibodies in the long-term clinical outcome of patients with Crohn's disease. Scand J Gastroenterol 2016;51:1081-6.

10. Ungar B, Levy I, Yavne Y, et al. Optimizing Anti-TNF- $\alpha$ Therapy: Serum Levels of Infliximab and Adalimumab Are Associated With Mucosal Healing in Patients With Inflammatory Bowel Diseases. Clin Gastroenterol Hepatol 2016;14:5507.e2. 
Figure 1. ROC curve analysis: adalimumab trough levels - disease activity at the end of follow up

Figure 2. ROC curve analysis: AAA trough levels - disease activity at the end of follow up 